I am grateful to Dr. F. H. Herbstein, of the National Physical Research Laboratory, Pretoria, for stimulating discussions. This communication is published with the permission of the South African Council for Scientific and Industrial Research.

$$
\text { J. F. DE WET }
$$

National Chemical Research Laboratory, South African Council for

Scientific and Industrial Research, Pretoria. July 12.

${ }_{1}$ Prelinger, O., Monatsh. Chem., 14, 353 (1893). ${ }^{2}$ de Wet, J. F., Angero. Chem., 67, 208 (1955). ' Dickinson, B. N., Z. Krist., A, 88, 281 (1934).

\section{Life-time of an Artificial Earth Satellite}

IN view of the recent widespread interest in the life-time of an artificial Earth satellite, a simple expres. sion is derived here which predicts the approximate life-time from initially measured values of the rate of change of height at apogee and the difference between the heights at apogee and perigee.

Let $h$ be the height at perigee and $h+2 h_{1}$ the height at apogee, both measured from the centre of the Earth. Using polar co-ordinates with $r$ as the distance from the centre of the Earth (the assumed origin) and $\theta$ the angle measured from perigee :

$$
r \sim \hbar+h_{1}(1-\cos \theta)
$$

and near perigee, where air resistance is much higher than elsewhere in all but the latest stage of the life of a satellite:

$$
r \sim h+\frac{1}{2} \theta^{2} h_{1}
$$

The impact of the air molecules at any point $r, \theta$ will cause no drop in height at that point but a maximum drop in height at the diametrically opposite point, so that the ratio of the height drop near perigee to that at apogee is :

$$
(1-\cos \theta) / 2 \sim \theta^{2} / 4
$$

Assuming that the air resistance causes a maximum height drop proportional to $\exp (-r / H) \mathrm{d} \theta$ over the angle $\mathrm{d} \theta$, where $H$, the atmospheric scale-height, is assumed much smaller than $h_{1}$, the total drop at perigee during one cycle is proportional to :

$$
\begin{aligned}
& \int_{-\pi}^{-\pi} \frac{1}{4} \theta^{2} \exp \left[\left(-h-\frac{1}{2} \theta^{2} h_{1}\right) / H\right] \mathrm{d} \theta \sim \\
& \frac{1}{8} \pi^{1 / 2}\left(h_{1} / 2 H\right)^{-3 / 2} \exp (-h / H)
\end{aligned}
$$

while similarly the drop at apogee is proportional to :

$$
\pi^{1 / 2}\left(h_{1} / 2 H\right)^{-1 / 2} \exp (-h / H)
$$

In view of the large decrease in the exponent of the exponential with increasing $\theta$, there will be little contribution to the integral over large values of $\theta$, and for simplicity the integrations were carried out from $\theta=-\infty$ to $\theta=+\infty$.

The ratio of the rate of decrease in height at perigee to that at apogee is then :

$$
\mathrm{d} h / \mathrm{d}\left(h+2 h_{1}\right) \sim \frac{1}{2} \mathrm{~d} h / \mathrm{d} h_{1}=\frac{1}{4} H / h_{1}
$$

Solving this differential equation :

$$
h_{1}=h_{10} \theta \mathrm{xp}\left[-2\left(h_{0}-h\right) / H\right]
$$

where $h_{10}$ and $h_{0}$ are the initial values of $h_{1}$ and $h$.
The rate of decrease in height at perigee is proportional to expression (1). Substituting $h_{1}$ from (4) one obtains :

$$
\mathrm{d} h / \mathrm{d} t \propto \exp [-4 h / H]
$$

the solution of which is

$$
t=t_{\lambda}\left\{1-\exp \left[-4\left(h_{0}-h\right) / H\right]\right\}
$$

The integration constant $t$, , which clearly is an approximation to the life-time of an Earth satellite, may be determined if the initial rate $h_{a}^{\prime}$ of losing height at apogee is known. Then the rate of loss of height at perigee is, from (3), given by $\frac{1}{4} h_{a}^{\prime} H / h_{1}$, which equals, from equation (6), for very small $t$ :

and therefore

$$
\left(h_{0}-h\right) / t \sim H /(4 t \lambda)
$$

$$
t_{\lambda}=h_{1} / h_{a}^{\prime}
$$

This simple expression means that the life-time of a satellite is approximately equal to the time in which the distance at apogee from the centre of the Earth, decreasing at its initial rate, would reach a value equal to the initial value of the semi-major axis of the orbit. The assumption $H \ll h_{1}$ is of course not valid in the last stages of a satellite's life. Expression (7) is therefore an under-estimate, but if $H<40 \mathrm{~km}$. the error is estimated to be less than 20 per cent.

From observations of the Doppler effect on the signals received from the $40.002 \mathrm{Mc}$./s. transmitter of the first Russian Earth satellite by the National Telecommunications Research Laboratory of the South African Council for Scientific and Industrial Research, the values $h_{1}=210$ statute miles and $h_{a}^{\prime}=$ $2.4 \mathrm{miles} /$ day (the latter derived from the measured rate of change of orbital period) have been obtained. From these a life-time $t_{\lambda}$ of 87 days is obtained as from October 20, 1957.

No radio measurements are available on the rocket casing, but from time differences between the satellite and the rocket casing published in the Press, a lifetime of about 56 days is obtained.

This communication is published with the permission of the South African Council for Scientific and Industrial Research.

\section{J. A. FEJER}

National Telecommunications Research Laboratory,

South African Council for Scientific and Industrial Research, P.O. Box 10319, Johannesburg. Oct. 25.

[See page 1392 of this issue of Nature.]

\section{Suppression of Bubbling in Boiling Refrigerants}

DURING recent studies of the properties of radicals trapped at low temperatures it has been found that the presence of bubbles in the body of a boiling refrigerant can be eliminated by introducing a stream of a gas which is comparatively insoluble in the refrigerant. In a typical experiment a small, unsilvered Dewar flask was half-filled with liquid nitrogen at atmospheric pressure, vigorous bubbling of the liquid being observed. When a jet of helium, hydrogen, or neon gas was blown over the liquid nitrogen at a 